\title{
Uso do Potencial de Sistemas de Realidade Virtual como Suporte para Aprender através de Processos Exploração, Observação e Construção Colaborativa de Conhecimento
}

\author{
Jorge Ferreira Franco ${ }^{1,2}$, Roseli de Deus Lopes ${ }^{1}$ \\ ${ }^{1}$ Laboratório de Sistemas Integráveis - Escola Politécnica da Universidade de São Paulo \\ (LSI/EPUSP) \\ Av. Prof. Luciano Gualberto, trav. 3, nº158 - 05508-970 - São Paulo - Brasil \\ ${ }^{2}$ Escola de Ensino Fundamental Ernani Silva Bruno - Secretaria Municipal de Educação \\ - Prefeitura Municipal de São Paulo (ESB - SME - PMSP) \\ Rua Interativa, 100 - 0202820 - São Paulo - Brasil \\ musiquarium 2000@yahoo.com,roselielsi.usp.br
}

\begin{abstract}
This paper presents a practical experience related to using virtual reality systems' potential based on Web3D accessible digital resources as support for learning and promoting individuals' lifelong education through exploring, observing and building knowledge processes, in a collaborative way, since the primary education teaching.
\end{abstract}

Resumo. Este artigo apresenta uma experiência relativa a usar o potencial de sistemas de realidade virtual com base em recursos digitais acessiveis da Web3D como suporte para aprender e promover educação continuada dos indivíduos, através de processos de explorar, observar e construir conhecimento, de modo colaborativo, desde o ensino fundamental.

\section{Introdução}

A crescente influência de tecnologias da informação e da comunicação (TIC) no cotidiano dos cidadãos e cidadãs amplia a relevância de propiciar formação continuada relativa ao conceito de alfabetização midiática e informacional (AMI) para educadores e estudantes, desde o ensino fundamental [Wilson et al., 2013].

Para [Wilson et al., 2013, p.16] o conceito de AMI engloba conhecimentos essenciais sobre as funções da mídia, das bibliotecas, da internet e de outros provedores de informação que são "reconhecidos como ferramentas essenciais para tomar decisões bem informadas". Tais conhecimentos permitem aos usuários se engajarem junto às mídias e aos canais de informação de modo significativo. Embasadas na AMI, as competências adquiridas podem equipar os indivíduos com raciocínio crítico, propiciando que demandem serviços de qualidade e contribuam com a formação de ambientes propícios para que as mídias e outros provedores prestem bons serviços.

Com melhor formação relativa ao conceito de AMI, os indivíduos podem utilizar o potencial de ambientes digitais bidimensionais (2D) e tridimensionais (3D) e da infraestrutura do ciberespaço para refletir sobre e transformar as maneiras de aprender, ensinar e conduzir pesquisa [Fox, Kelly, Patil, 2010]. Incluindo, se beneficiarem do uso 
de ambientes digitais 2D/3D e tecnologias afins como suportes para seu desenvolvimento de conhecimento e cognitivo [Sharp, Rogers e Preece, 2007].

Há mais acessibilidade dos cidadãos a software, hardware, sistemas midiáticos e conteúdos complexos. Portanto, é necessário promover processo de desenvolvimento de conhecimento e aprimorar a competência técnica dos indivíduos continuamente para que compreendam e usem o potencial de sistemas midiáticos complexos com aprofundamento e também se tornem produtores de conteúdo digital, por exemplo, através de utilizarem ambientes digitais 3D e tecnologias afins [Franco e Lopes, 2012].

Este artigo apresenta uma experiência de uso do potencial de sistemas de realidade virtual (RV) com base em recursos digitais acessíveis da Web3D como suporte para promover aprendizagem de indivíduos, através de processos de explorar, observar e construir conhecimento, de modo colaborativo, no ensino fundamental.

Contribuições qualitativas deste trabalho englobam promover engajamento de estudantes em processos reflexivos e interativos de leitura e escrita tradicional, midiática e visual com base em atividades de programar computador, produzir e visualizar informação em tempo real, e estimular uso complexo de recursos multimídia na escola e em casa, para aprimorar habilidades cognitivas e competência técnica com inovação.

Incluindo, instigar raciocínio crítico dos indivíduos para compreender e usar tecnologias e meios de produção contemporâneos de modo inter-relacionado com aplicar conhecimentos de várias ciências que compõem o currículo escolar, tanto quanto promover atividades educativas continuadas, sustentáveis e desenvolvidas com maior autonomia pelos cidadãos [Franco e Lopes, 2012, 2013; Wilson et al., 2013].

\section{Abordagem Pedagógica com Suporte do Conceito de AMI}

Conforme Wilson e colegas [2013, p.76] o conceito de AMI suporta aplicar várias abordagens pedagógicas nas atividades de ensino-aprendizagem. Entre elas, está a abordagem produtiva, "que implica em aprendizagem pela prática, que é um importante aspecto da assimilação de conhecimentos do século XXI. Os estudantes devem ser levados a explorar a aprendizagem em um nivel mais profundo e significativo".

$\mathrm{Na}$ abordagem produtiva, os estudantes/educadores têm oportunidades de protagonizar, expressando suas ideias, criatividade e perspectivas através de recursos midiáticos contemporâneos e tecnologias afins, como computadores e celulares; linguagens de programação; e arquivos multimídia acessíveis. Deste modo, a produção de conteúdo de mídia (áudio, vídeo, gráficos $2 \mathrm{D}$ e 3D, textos) permite "uma imersão na aprendizagem por meio da exploração e da ação" [Wilson et al. 2013, p.76].

Um modo de promover imersão, no processo de aprendizagem, é estimular capacidades cognitivas e competência técnica dos indivíduos com base também em intervenção pedagógica dialógica [Freire, 2011] e interações humano computador (IHC) [Netto, 2010]. E aplicar estratégia de manipulação direta de linguagens de programação de computadores e sistemas de visualização de informação acessíveis do ciberespaço, para que os indivíduos compreendam como usá-los, via procedimento de construção (design, produção e visualização de conteúdo digital 2D e 3D) [Franco e Lopes 2012].

A partir da inter-relação entre o conceito de AMI, abordagem pedagógica produtiva, intervenção pedagógica dialógica, IHC e processos de aprendizagem 
suportados por meios contemporâneos de produção, o conhecimento, as habilidades cognitivas e competência técnica dos indivíduos tendem a ser construídos e aplicados de maneira consciente e aprofundada por meio de observar, explorar, agir e refletir.

Pois, segundo Freire [2011, p. 42-43] "o conhecimento não se estende daquele que se julga sabedor até aqueles que se julga não saberem; o conhecimento se constitui nas relações homem mundo, relações de transformação, e se aperfeiçoa na problematização crítica dessas relações". Para refletir com os indivíduos questões de ordem técnica, é preciso que, para eles o tema mencionado já constitua "um percebido destacado em si". Daí, os indivíduos captem as relações interativas entre o percebido destacado e outras dimensões da realidade. "Isso, demanda um esforço não de extensão, mas de conscientização que, se bem-realizado, permite aos individuos se apropriarem criticamente da posição que ocupam com os demais no mundo" [Freire, 2011, p.43].

\section{Realidade Virtual, Tecnologias da Web3D, Instrumentos Técnicos}

Linguagens de programação e sistemas de visualização de informação acessíveis do ciberespaço são instrumentos técnicos que compõem vários ecossistemas de realidade virtual, entre eles, os com base em recursos/tecnologias digitais acessíveis da Web3D.

Eles possibilitam produzir um código/programa, via linguagens, como a Virtual Reality Modeling Language (VRML) e a Extensible 3D Language (X3D) [Brutzman e Daly, 2007] e visualizar sua representação simbólica com suporte de navegadores, como o [InstantReality $\left.{ }^{\mathrm{TM}}, 2013\right]$ e [FreeWRL $\left.{ }^{\mathrm{TM}}, 2013\right]$. E permitem desenvolver várias aplicações educacionais e de treinamento com base em visualização de informação para suportar aprendizagem de conceitos científicos [Cardoso e Lamounier, 2006].

Em trabalhos prévios foram utilizados instrumentos técnicos (navegadores da Web3D) referentes às linguagens VRML e X3D [Franco e Lopes, 2012]. Porém, devido a uma política de administração de segurança dos recursos de informática institucional, no laboratório da escola foi reduzido o acesso e autonomia para instalar softwares, ainda que sejam livres nos 20 computadores utilizados pelos estudantes, exceto, no computador do professor orientador de informática educativa (POIE).

Entretanto, através de processo de educação continuada do autor1, houve acesso ao X3DOM, um arcabouço/'framework' experimental, de código aberto e 'runtime' (dinâmico), que suporta investigações de grupos como Web3D e W3C, a respeito da integração entre Hypertext Markup Language (HTML5) e conteúdo 3D declarativo. Este arcabouço permite que se incluam elementos X3D em um documento com formato HTML5. Não há necessidade de usar um 'plugin' (software para visualização dos elementos 3D), como é o caso da especificação X3D. O arcabouço X3DOM integra, na base de desenvolvimento de seu ecossistema, tecnologias como a X3D, HTML5, JavaScript e Python. Possibilita criar conteúdo digital 2D e 3D dinâmico através de combinar scripts HTML5 e X3D e o visualizar com navegador da internet, com suporte da tecnologia Canvas3D. Por exemplo, os navegadores Google Chrome ${ }^{\mathrm{TM}}$, FireFox ${ }^{\mathrm{TM}} \mathrm{e}$ Internet Explorer ${ }^{\mathrm{TM}}$ em uma versão que suporte a tecnologia WebGL [X3DOM, 2013].

Embora não seja necessário instalar um 'plugin' para usar o arcabouço X3DOM, as experiências na escola (ESB) e a literatura (ver documentação de desenvolvimento no sítio do [X3DOM, 2013]) indicam que quando não se está on-line, não se consegue 
visualizar o conteúdo 3D no computador local, pois o script do programa busca informações e funcionalidades para visualização de conteúdo $3 \mathrm{D}$ em servidor externo. Entretanto, conforme documentação [X3DOM, 2013], havendo habilidade técnica e acesso ao sistema de administração de segurança de rede, é possível instalar os 'softwares livres' necessários para visualização off-line em um servidor institucional.

Outros instrumentos técnicos utilizados para desenvolver conteúdo digital 3D com uso do X3DOM são: um editor de texto como o (bloco de notas) e um computador contendo processador e ou placa de vídeo com características técnicas de processar WebGL. Após inserir o script do programa no bloco de notas; salvar como: ("nome do arquivo.html'); para abri-lo e visualizar sua representação simbólica em 3D, abra a pasta onde o 'arquivo.html' foi salvo, clique duas vezes sobre ele, ou clicando o lado direito do mouse (em sistema Windows), aparece um 'menu', com a inscrição abrir com, então escolha um navegador da internet compatível com a tecnologia WebGL.

\section{Práticas de uso de realidade virtual com base em tecnologias da Web3D}

Ambientes digitais 3D referentes ao framework X3DOM podem ser editados em computador local e/ou on-line com inserção e/ou programação de código e visualização em tempo real, por exemplo, através do site (WWW.blogger.com). Um exemplo, figura 1, é um blog (http://856585.blogspot.com.br/) de um dos participantes do projeto de estudos colaborativos com doze estudantes de como usar o potencial de sistemas de RV com base em recursos digitais acessíveis da Web3D, na escola Ernani Silva Bruno (ESB), situada em Parada de Taipas, um bairro da periferia da cidade de São Paulo.
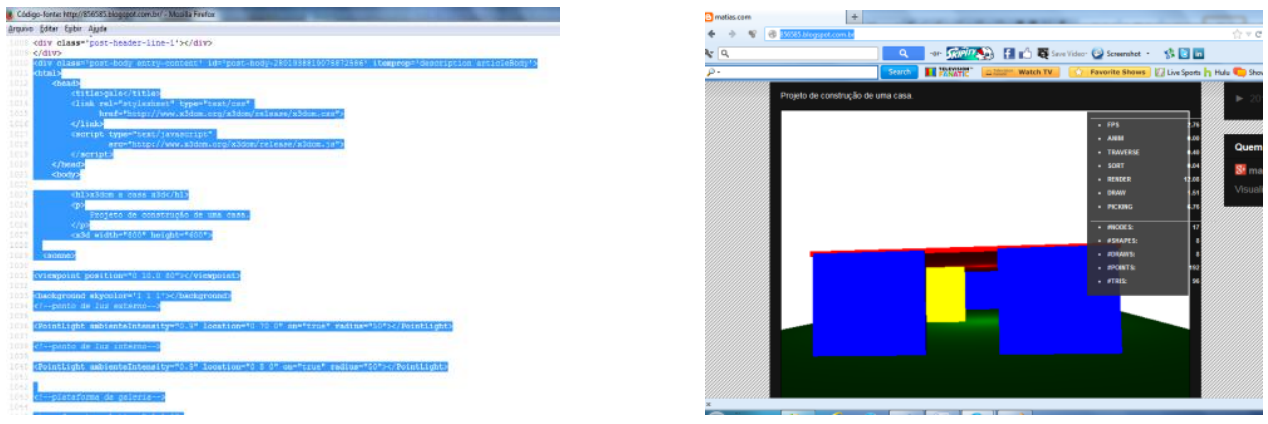

Figura 1. Lado esquerdo parte de código fonte X3DOM integrando HTML e X3D. Lado direito, visualização da representação simbólica do código via objeto 3D

Com suporte da abordagem pedagógica descrita na seção 2, o uso do arcabouço X3DOM contribui para estimulo processual de capacidades cognitivas e competência técnica de estudantes e educadores, desde o segundo semestre de 2012. Este trabalho utiliza a estratégia de promover oficinas interativas, de pequena escala, que têm base em manipulação direta de arquivos X3DOM e criação de objetos digitais 3D de modo interrelacionado com diversos conceitos científicos do currículo escolar. Esta estratégia tende a facilitar que os participantes reflitam, decomponham e compreendam a simplicidade e a complexidade de conhecimentos diversos que são entrelaçados e compõem ambientes digitais 3D (AD3D), como exemplifica a figura 1, e publicação de vídeo demo com estudantes produzindo conteúdo 3D, no sítio [X3DOM, 2013].

Conteúdos explorados durante os experimentos e observações de criação de AD3D estão interligados com o contexto de promover aprendizagem formal e informal. 
Para Sharp, Rogers e Preece [2007] habilidades cognitivas, como as de ler, falar, escrever, memorizar, perceber e reconhecer, atenção, resolver problemas, raciocinar, tomar decisão e aprender são processos inter-relacionados. Por exemplo, combinar a habilidade cognitiva de aprender com a competência técnica de programar pode estimular leitura e escrita a partir de reutilizar e/ou programar um trecho de código $\mathrm{X} 3 \mathrm{DOM}$ e visualiza-lo em tempo real, como na figura 1. Utilizando a estratégia de 'aprender através de fazer', indivíduos manipulam diretamente uma interface gráfica e podem se beneficiar de possibilidade de aprendizagem ativa com suporte de interação exploratória 'tentativa e erro' embasada em funcionalidades, como as de 'fazer e desfazer' de um programa de modo combinado com conceitos científicos do currículo.

Esta combinação suporta refletir sobre como usar AD3D para explorar conceitos relativos a números positivos e negativos, plano Cartesiano (X, Y, Z) e cognição espacial de modo inter-relacionado com o comando <transform translation="-14.5 5. $0^{\prime \prime}>$. Aprender inglês, com consciência de possibilidade de aplicar a língua, no contexto de promover desenvolvimento técnico dos indivíduos. Implementar e visualizar conceitos de textura e iluminação, estudados em artes, como na figura-1. Simular ambientes, exemplo galerias virtuais, construindo-os com suporte de planos de estudos pré-desenvolvidos, através de pesquisas e interações com outros materiais, como livros, papel, lápis e régua para esboçar o dimensionamento de um ambiente 3D, figura 2 .
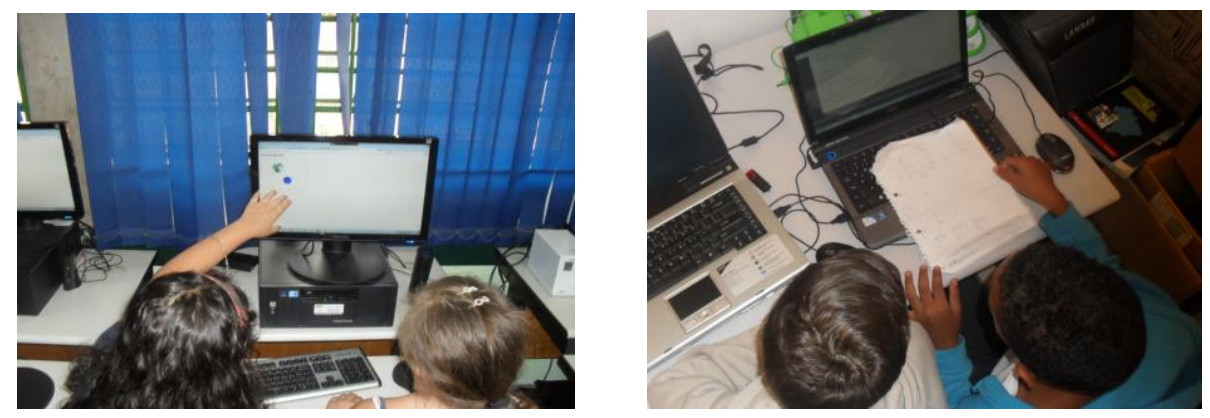

Figura 2. Momentos de aprendizagem colaborativa com suporte de recursos técnicos da Web3D. À esquerda na escola. À direita na FEBRACE

As atividades educativas propiciam momentos de aprendizagem colaborativa e continuada para estudantes e educador envolvendo recursos informacionais e ambientes virtuais e reais diversos, como os do laboratório de informática da escola, casas dos estudantes e participação na Feira Brasileira de Ciências e Engenharia figura 2.

Para além do espaço escolar, o uso de tecnologias da Web3D gerou uma oficina de formação para professores da rede pública municipal de São Paulo. Durante evento promovido pela Diretoria Regional de Ensino de Pirituba, em 2012, sob a coordenação do autor1, educadores de ensino fundamental e médio manipularam arquivos X3DOM, se engajando em processo de programação de computadores e visualização de informação com conteúdo 3D, em tempo real, similar aos das figuras 1 e 2 .

\section{Considerações Finais}

Com base na necessidade de estimular alfabetização midiática e informacional e de instigar constante construção de saberes dos cidadãos envolvendo conhecimento técnico e prático referentes a explorar e criar ambientes digitais $3 \mathrm{D}$. 
Neste artigo são apresentados conceitos pedagógicos e técnicos tanto quanto exemplos empíricos, no contexto de ensino fundamental, que embasam a prática de uso do potencial de sistemas de realidade virtual como suporte para aprender através de processos exploração, observação e construção colaborativa de conhecimento. Com tais conhecimentos e habilidades midiáticas e informacionais, de modo formal e informal, os cidadãos comuns e educadores "terão capacidades aprimoradas de empoderar os estudantes em relação a aprender a aprender, a aprender de maneira autônoma e a buscar educação continuada" [Wilson et al., 2013, p.17].

\section{Agradecimentos}

Agradecemos o apoio dos estudantes e educadores que contribuem para a continuidade deste projeto. Que sejam todos muito abençoados.

\section{Referencias}

Brutzman, D. e Daly, L. (2007) Extensible 3D Graphics for Web Authors, China, Morgan kaufman.

Cardoso, A. e Lamounier, E. (2006). A realidade virtual na educação e treinamento. In: Tori, R. kirner, C. e Siscouto, R. (Org) Fundamentos e tecnologia de realidade virtual e aumentada. Brasil: Editora SBC.

Fox, M. R., Kelly, H. e Patil, S. (2010) Medulla: A cyberinfrastructure-enabled framework for research, teaching and learning with virtual worlds. In: Bainbridge, W. W. (Ed.) Online Worlds: convergence of the real and the virtual. England: Springer.

FreeWRL, (2013) http://freewrl.sourceforge.net/download.html, Acesso em 07/10/2013

Freire, P. (2011) Extensão ou comunicação? Brasil: Paz e Terra.

InstantReality (2013), http://www.instantreality.org/story/what-is-it/, Acesso em $11 / 08 / 2013$

Netto, A. A. O. (2010) IHC e a engenharia pedagógica, Brasil: Visual Books.

Franco, J. F. e Lopes, R. D. (2013) Using X3DOM for Promoting Students' Digital and Visual Literacy at K-12 Education: a Use Case, In: Proceedings of Web3D 2013, June 20 - 22, 2013, San Sebastian, Spain: ACM. 2013

Franco, J. F. e Lopes, R. D. (2012) Developing an interactive knowledge-based learning framework with support of computer graphics and web-based technologies for enhancing individuals' cognition, scientific, learning performance and digital literacy competences, IN: Mukai, N. (Ed), Computer Graphics, Croatia: Intech, 2012. http://www.intechopen.com/books/computer-graphics, Acesso em 30/10/2013

Wilson, C. et al. "Alfabetização midiática e informacional: currículo para a formação de professores”, Brasília, 194p. http://pt.scribd.com/doc/157680302/Alfabetizacaomidiatica-e-informacional-Curriculo-para-formacao-de-professores-UNESCO, Acesso em 30/08/2013

X3DOM (2013) Instant 3D the HTML way, http://www.x3dom.org/?paged=3, Acesso em $07 / 10 / 2013$ 